\title{
Raster Thomson scattering in large-scale laser plasmas produced at high repetition rate
}

\author{
M. Kaloyan, ${ }^{1, \text { a) }}$ S. Ghazaryan, ${ }^{1}$ C. G. Constantin, ${ }^{1}$ R. S. Dorst, ${ }^{1}$ P. V. Heuer, ${ }^{2}$ J. J. Pilgram, ${ }^{1}$ D. B. Schaeffer, ${ }^{3}$ \\ and C. Niemann ${ }^{1}$ \\ 1) Department of Physics and Astronomy, University of California Los Angeles, Los Angeles, CA 90095, \\ USA \\ ${ }^{2)}$ Laboratory for Laser Energetics, University of Rochester, 250 East River Road, Rochester, NY 14623, \\ USA \\ ${ }^{3)}$ Department of Astrophysical Sciences, Princeton University, Princeton, NJ 08540, USA
}

\begin{abstract}
We present optical Thomson scattering measurements of electron density and temperature in a large-scale $(\sim 2 \mathrm{~cm})$ exploding laser plasma produced by irradiating a solid target with a high energy (5-10 J) laser pulse at high repetition rate $(1 \mathrm{~Hz})$. The Thomson scattering diagnostic matches this high repetition rate. Unlike previous work performed in single shots at much higher energies, the instrument allows point measurements anywhere inside the plasma by automatically translating the scattering volume using motorized stages as the experiment is repeated at $1 \mathrm{~Hz}$. Measured densities around $4 \times 10^{16} \mathrm{~cm}^{-3}$ and temperatures around $7 \mathrm{eV}$ result in a scattering parameter near unity, depending on the distance from the target. The measured spectra show the transition from collective scattering close to the target to non-collective scattering at larger distances. Densities obtained by fitting the weakly collective spectra agree to within $10 \%$ with an irradiance calibration performed via Raman scattering in nitrogen.
\end{abstract}

\section{INTRODUCTION}

Thomson scattering (TS) is a powerful first-principles, noninvasive plasma diagnostic that has been applied extensively to characterize dense millimeter-scale laser produced plasmas (LPP) encountered in inertial confinement fusion and highenergy density physics research $\frac{112}{1}$. There has recently been increasing interest in understanding the less explored latetime evolution of exploding laser-plasmas. These are used, for example, to study the physics of space and astrophysical phenomena in appropriately scaled laboratory experiments 3 , for thin film deposition of materials ${ }^{4}$, and for laser-ablation propulsion research ${ }^{5}$. At these late times, the LPP expands to much larger scales of centimeters ${ }^{6}$ to meters ${ }^{7}$, while its density and temperature drop significantly. In order to apply TS in these plasmas, one has to overcome challenges associated with the small scattering cross section, similar to those encountered when scattering off magnetic confinement fusion plasmas $^{8} 11$ or low temperature plasmas $12+16$. These include the need for stray light mitigation using a notch filter, an absolute irradiance calibration of the detection branch, and photon averaging over hundreds of laser shots.

We have developed a high-repetition rate TS diagnostic to provide model-independent volumetric measurements of electron density and temperature in large-scale LPPs produced at high shot rate $(1 \mathrm{~Hz})$ anywhere inside the plasma, with high spatial $(<1 \mathrm{~mm})$ and temporal $(4 \mathrm{~ns})$ resolution. Most previous TS systems for single shot laser-plasma experiments have produced measurements at one single point in space and time 17 . Some experiments have employed imaging spectrometers to obtain 1D-spatially resolved data at one single time ${ }^{18 / 19}$, while others have used streaked spectrometers to obtain plasma parameters at a single spatial point as a function of time ${ }^{1}$. By contrast, the instrument described here uses

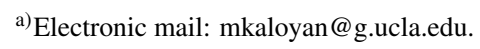

motorized stages to auto-align and translate the scattering volume between laser shots. Spatially resolved data is obtained by automatically scanning the scattering volume across a predefined spatial pattern, as the experiment is repeated thousands of times. Automated alignment and translation of the scattering volume are made possible by an optical design that optimizes the trade-offs between alignment sensitivity on the one hand and optical extent and photon count on the other. This capability to collect volumetric measurements of plasma density and temperature is important for a new generation of high-repetition-rate high-energy laser facilities 20 -23.

In section III we present a detailed description of the instrument, its optical design, and the notch filter for stray light rejection. First proof-of-concept scattering measurements in a relatively unexplored large-scale $(\sim 2 \mathrm{~cm})$, high-energy $(5-$ $10 \mathrm{~J})$ LPP are presented in section IV] For now these experiments were limited to measurements along the blow-off axis due to the low probe beam energy $(50 \mathrm{~mJ})$. Electron densities obtained by fitting the weakly collective spectra agree with an absolute irradiance calibration via Raman scattering (section III to within $10 \%$, which is well within the accuracy of the density measurement.

\section{EXPERIMENTAL SETUP}

A schematic of the experimental setup is shown in Fig. 11. The plasma is created by ablating a solid plastic target with a high energy laser pulse. The laser used to heat the target is a high-repetition rate flashlamp-pumped Nd:glass zig-zag path slab laser with wavefront correction using a stimulated Brillouin scattering phase conjugate mirrol ${ }^{24}$. The laser delivers an energy up to $10 \mathrm{~J}$ in a near diffraction limited beam at 1053 $\mathrm{nm}$ with a pulse duration of $14 \mathrm{~ns}$ at a repetition rate of $1 \mathrm{~Hz}$. The output energy is stable to within 5\%, and the pulse shape and pointing are stable to within $1 \%$. The beam is focused onto a cylindrical high density polyethylene $\left(\mathrm{C}_{2} \mathrm{H}_{4}\right)$ plastic target mounted on a $2 \mathrm{D}$ stepper motor drive. The target drive 
is synchronized with the laser, and is rotated and translated in a helical pattern to provide a fresh surface for each shot ${ }^{25}$. The beam is incident on the target at an angle of $34^{\circ}$ relative to the target normal and is focused to an intensity around $\sim 10^{12}$ $\mathrm{W} / \mathrm{cm}^{2}$ using an $\mathrm{f} / 25$ spherical lens with a focal length of 1.0 $\mathrm{m}$. The LPP explodes normal to the target surface regardless of the angle of incidence. A $200 \mathrm{l} / \mathrm{s}$ turbo-pump stabilizes the vacuum pressure during $1 \mathrm{~Hz}$ laser ablation at around $7 \times 10^{-3}$ torr.

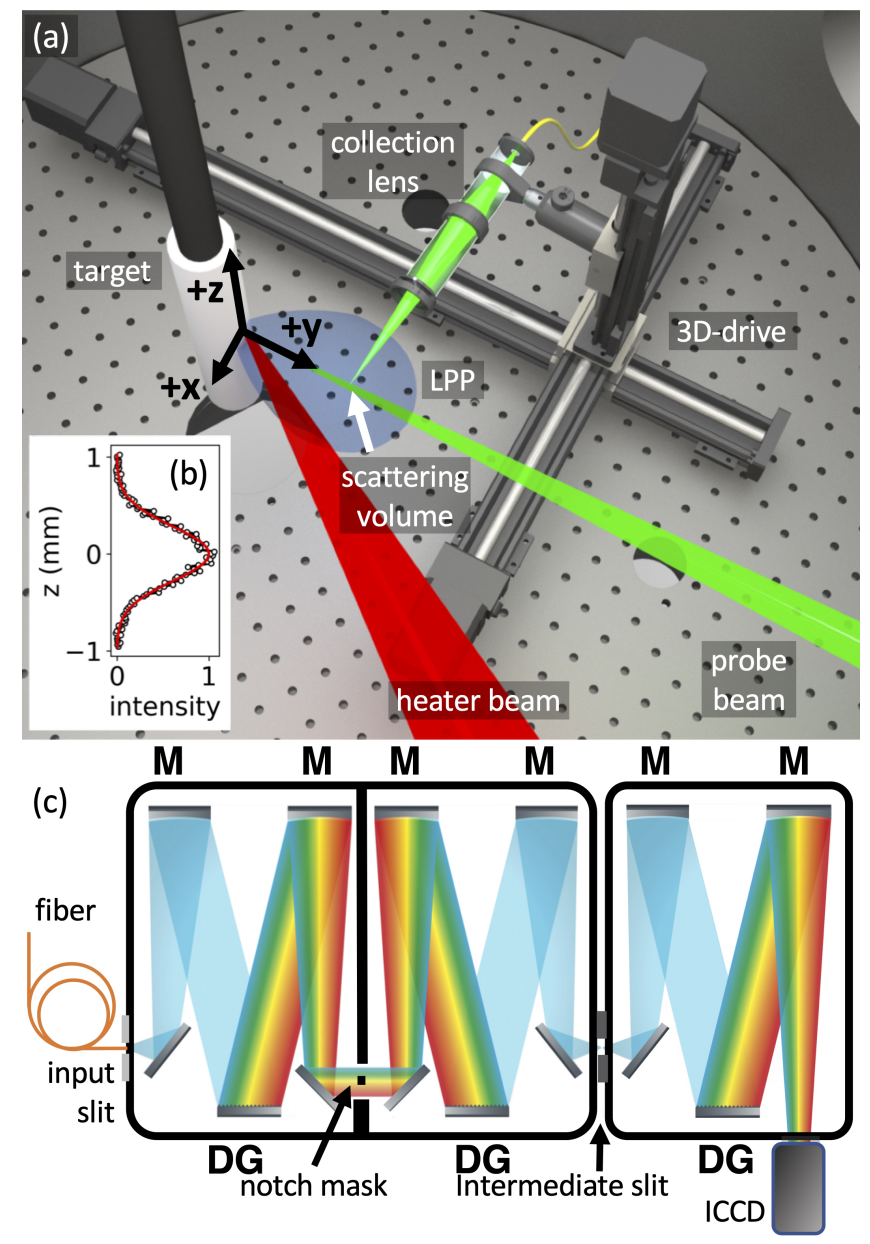

FIG. 1. (a) Schematic layout of the experiment showing the target and beam configuration and the collection branch on the 3D-drive. (b) The scattering volume is automatically aligned by scanning the lens and fiber along $\mathrm{z}$ while monitoring the Raman scattering intensity off air. (c) A diagram showing the light path through the triple grating spectrometer used for stray light suppression shows the notch mask between the double-subtractive stages.

A separate pulse from a second diode-pumped Nd:YAG laser is used as the TS probe beam. This beam delivers an energy of $E_{\text {pulse }}=50 \mathrm{~mJ}$ in a $\tau_{L}=4 \mathrm{~ns}$ pulse at the second harmonic $\left(\lambda_{i}=532 \mathrm{~nm}\right)$, and enters the target chamber through an anti-reflection coated flat vacuum window and disposable blast shield exactly along the plasma blow-off axis $(-\hat{y})$. A slow (f/150) spherical lens with a focal length of $75 \mathrm{~cm}$ is used to focus the probe beam to a point $3 \mathrm{~cm}$ from the target surface along $\hat{y}$. This configuration produces a cylin- drical "pencil" beam of constant $0.4 \mathrm{~mm}$ diameter over the $6 \mathrm{~cm}$ Rayleigh length in front of the target. The probe beam is synchronized to the heater pulse including a variable delay between 50-300 ns, and is linearly polarized along the zaxis. The probe beam terminates on the target, creating copious amounts of stray light as well as a secondary laser-plasma plume. However, this secondary laser plasma reaches the scattering volume hundreds of ns after the time of the scattering measurement, and consequently does not affect our results.

Scattered light is collected perpendicular to the probe beam with a $5.0 \mathrm{~cm}$ focal length, $12 \mathrm{~mm}$ diameter aspheric collection lens into an $\mathrm{f} / 20$ collection cone, and is focused at $\mathrm{f} / 5.3$ into a single $200 \mu \mathrm{m}$ core optical fiber with a numerical aperture of NA $=0.22$. The $40 \mathrm{~m}$ long fiber is coupled to the input slit of the spectrometer via a fiber vacuum feed-through. The scattering volume is defined by the intersection of the probe beam and the projection of the magnified fiber core cross section onto the beam. The collection branch is designed to project the fiber core with a $3.8 \times$ magnification onto the probe beam, so that the $0.7 \mathrm{~mm}$ diameter fiber projection exceeds the probe beam size by a factor of $\sim 2$. At first glance it would seem that the slow $\mathrm{f} / 20$ collection cone would result in more than an order of magnitude loss in collection efficiency, compared to a much faster design often used (f/4). However, it should be considered that the total number of collected photons is proportional to the source optical extent $G^{\prime}=A^{\prime} \cdot \Omega^{\prime}$, where $A^{\prime}$ is the source area, and $\Omega^{\prime}=\pi /\left(2 \cdot f \#^{\prime}\right)^{2}$ is the collection cone solid angle, where $f \#^{\prime}=20$ is the collection cone f-number. The larger f-number results in a larger source area because the magnification of the fiber onto the probe beam $|M|=f \#^{\prime} / f \#$ increases linearly with $f \#^{\prime}$, while the f-number into the fiber $f \#=5.3$ is fixed. When scattering off a pencil beam with a diameter smaller than the fiber projection, $G^{\prime}$ scales inversely proportional to the $\mathrm{f}$-number. The parameters used here result in $G^{\prime}=8.5 \times 10^{-4} \mathrm{rad} \cdot \mathrm{mm}^{2}$, which is still $70 \%$ of the theoretical upper limit set by the optical extent $G$ of the spectrometer f-number and fiber area. This acceptable loss in photons significantly reduces alignment sensitivity, making it possible to automatically translate the scattering volume along the probe beam using motorized stages.

The collection lens, along with a fused silica blast shield, 3 $\mathrm{mm}$ diaphragm, and fiber launch are housed in a light-tight $25 \mathrm{~mm}$ diameter tube. The assembly is installed on a 3D stepper-motor controlled probe drive inside the vacuum vessel, which can position the scattering volume anywhere inside a $10 \times 10 \times 10 \mathrm{~cm}^{3}$ volume in front of the target, with $5 \mu \mathrm{m}$ resolution and bidirectional repeatability. Raman scattering off of air at atmospheric pressure is used to automatically align the collection branch to the probe beam. Fig. 1p shows the measured Raman intensity as a function of vertical lens position $(\hat{z})$. The data was obtained using a camera exposure of $1 \mathrm{~s}$ with the probe laser pulsing at $50 \mathrm{~Hz}$. The Gaussian fit to the measured profile (red line) has a width of $0.7 \mathrm{~mm}$ (FWHM), which corresponds to the convolution of fiber core projection and the $0.4 \mathrm{~mm}$ diameter probe beam. The center of the fit marks the optimum lens alignment. The vertical beam position is mapped out automatically this way as a function of distance from the target $(\hat{y})$. Raman scattering measurements 
in air confirmed that the collection efficiency remains constant when translating the scattering volume along the probe beam $(\hat{y})$. Similarly, collection efficiency does not vary when translating the collection lens transversely to the probe $(\hat{x})$ over a range of $\pm 1 \mathrm{~cm}$, as expected given the large mismatch between fiber projection and beam diameter, and the slow f/20 collection cone.

A triple-grating spectrometer (TGS) with a spatial filter is used to significantly reduce stray light without eliminating the TS signal. The spectrometer used is a $0.5 \mathrm{~m}$ focal length f/4 Czerny-Turner type imaging spectrometer with three 1200 grooves/mm holographic gratings. Scattered light enters the spectrometer through a $200 \mu \mathrm{m}$ input slit, is collimated and imaged by internal mirrors, and passes through a notch filter and $2.0 \mathrm{~mm}$ intermediate slit before reaching the detector. The first two gratings are used in subtractive dispersion and serve as a notch filter to reject stray light. The last grating disperses the light onto the detector. Toroidal mirrors image the input slit onto the notch filter, intermediate slit and detector plane. As illustrated in Fig. 18, the notch filter is placed in between the double-subtractive spectrometers. We used an $0.75 \mathrm{~mm}$ wide and $50 \mu \mathrm{m}$ thick stainless steel mask, which blocks a wavelength range of of $1.5 \mathrm{~nm}$. The total transmission through the spectrometer without the notch is measured to be $25 \%$ and is mostly determined by the reflectivity of the three holographic aluminum-coated gratings blazed at $500 \mathrm{~nm}$. The spectral resolution was measured to be $0.22 \mathrm{~nm}$ over a wavelength range of $19.4 \mathrm{~nm}$.

Spectra are recorded using an image intensified charge couple device (ICCD) directly mounted to the output of the third spectrometer stage. The camera has a generation III photocathode with a quantum efficiency of $50 \%$ at $532 \mathrm{~nm}$. The micro-channel plate (MCP) is gated at $4 \mathrm{~ns}$ and synchronized to the 4 ns probe pulse with a jitter of less than 100 ps. We kep the MCP gain fixed at the maximum of of $\mathrm{G}=233$, and used $2 \times 2$ hardware binning for all images. Even with full gain and binning, the pixel count is a small fraction of the 16-bit maximum and well within the linear response range. The spectra are further binned over 250 vertical pixels along the fiber and slit and over two pixels horizontally into 256 total bins with $0.076 \mathrm{~nm} / \mathrm{bin}$. The $20 \mathrm{e}^{-} /$pixel readout noise with the ICCD cooled to $-20^{\circ} \mathrm{C}$ is negligible with this binning when averaging multiple shots.

Carbon plasma self emission lines from the LPP are also present in the spectrum that can have intensities higher than the scattering signal. To eliminate these lines, a plasma-only background spectrum recorded without the probe is subtracted from each scattering spectrum. An equal number of TS images and plasma-only background images are interleaved by creating the LPP at $1 \mathrm{~Hz}$ but pulsing the probe laser on only every other shot $(1 / 2 \mathrm{~Hz})$. While the plasma emission and residual stray light background can be subtracted from the data, their shot noise can not. The total noise is determined by the shot noise of both the TS signal and plasma background. Integrating over $n$ laser shots increases the total number of TS counts and background counts equally to $n \cdot N_{T S}$ and $n \cdot N_{b g}$, respectively. Assuming Poisson statistics, the noise of each signal component increases with the number of shots as $\sqrt{n}$, as does the signal to noise ratio (SNR)

$$
\mathrm{SNR}=\frac{\mathrm{n} \cdot \mathrm{N}_{\mathrm{TS}}}{\sqrt{\mathrm{n} \cdot \mathrm{N}_{\mathrm{TS}}+\mathrm{n} \cdot \mathrm{N}_{\mathrm{bg}}}} \sim \sqrt{\mathrm{n}} .
$$

In order to increase SNR we averaged between 100-400 shots (in addition to an equal number of background shots) for each spectrum, depending on the density and the amount of plasma emission.

Fig. 2 shows the spectrum of stray light, measured without the notch, caused by the probe laser scattering off the target and other surfaces inside the chamber. Since the laser line width is negligible, the profile is a measure of the instrument function. The spectrum is averaged over 300 shots to increase SNR in the wings. The instrument profile is the convolution of the Gaussian contribution due to the aberrated slit width, and the Lorentzian profile caused by diffraction of the three gratings. The notch reduces the intensity of the central $532 \mathrm{~nm}$

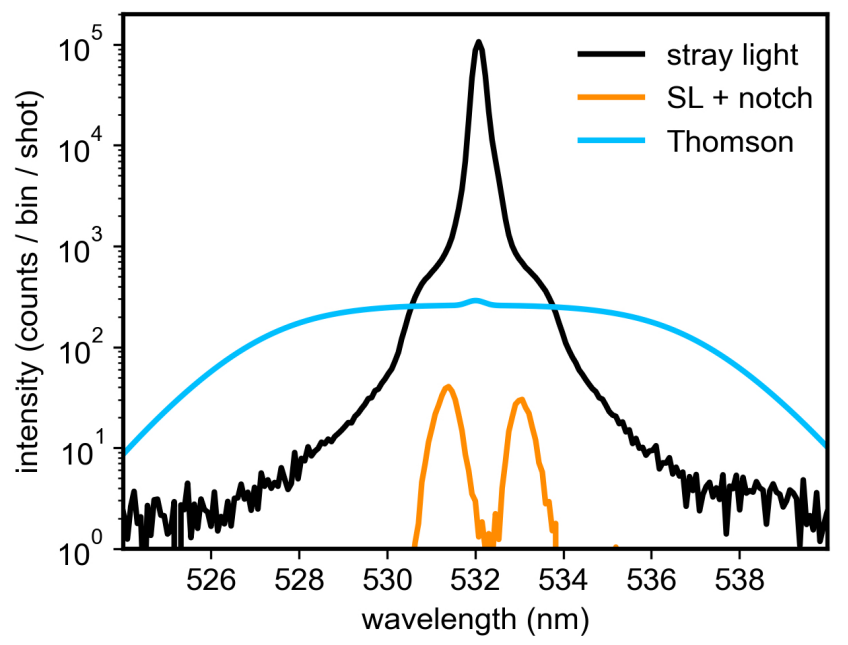

FIG. 2. The stray light spectrum (black) is used to measure the instrument function. The profile shows a Gaussian peak with $0.22 \mathrm{~nm}$ FWHM and the Loretzian wings caused by diffraction off the gratings. A simulated and absolutely scaled TS spectrum for $7 \mathrm{eV}$ and $4 \times 10^{16} \mathrm{~cm}^{-3}$ is shown for comparison (blue line). At $532 \mathrm{~nm}$ the stray light is three orders of magnitude higher in amplitude than the TS signal. The orange curve shows the leakage of the stray-light through the $1.5 \mathrm{~nm}$ notch filter. The notch reduces the central stray light $\left(\lambda_{i}\right)$ by five orders of magnitude and the wings by three. The notch filter suppresses the stray light to well below $1 \%$ of the TS signal except for wavelengths close to $\lambda_{i}$.

wavelength by five orders of magnitude (orange line) and the wings by three orders of magnitude. The notch reduces the intensity of the wings also outside its $1.5 \mathrm{~nm}$ width, since it removes $532 \mathrm{~nm}$ light before it diffracts off the final two gratings. The total extinction ratio of the notch, integrated over all wavelength was $2.0 \times 10^{4}$, equivalent to an optical density of OD 4.3. A simulated and absolutely scaled TS spectrum for $4 \times 10^{16} \mathrm{~cm}^{-3}$ and $7 \mathrm{eV}$ is shown for comparison (blue line). Without the notch, the stray light exceeds the scattering signal by three orders of magnitude, and the stray light amplitude in the wings is no less than $10 \%$ of the TS intensity across all 
wavelengths. The notch filter suppresses the stray light to well below $1 \%$ of the TS signal except for wavelengths closest to $\lambda_{i}$.

\section{ABSOLUTE SPECTROMETER CALIBRATION}

For laser light scattering off microscopic particles, the total number of counts (photo-electrons) measured by a detector is

$$
N=\underbrace{\overbrace{\tau_{L} \cdot I_{L}}^{\text {laser }} \overbrace{\Delta V \Delta \Omega}^{\text {collection }} \cdot \overbrace{\mu \eta}^{\text {optics }}}_{k} \cdot n \cdot \frac{d \sigma}{d \Omega},
$$

where $n$ is the density of scattering particles and $d \sigma / d \Omega$ is the differential cross section. The experimental throughput parameter $k$ depends on the probe laser, the collection optics and scattering volume, transmission through all optical components, and the camera response. Here $I_{L}$ is the probe laser intensity in the scattering volume $\Delta V$, and $h v_{i}$ is the energy of one laser photon. Only a fraction of the photons scattering into the solid angle $\Delta \Omega$ make it to the detector, depending on the optical transmission $\mu$ through the lens, optical fiber, and spectrometer. Lastly, photons are converted to photo-electrons with quantum efficiency $\eta$, which are multiplied by the MCP with gain $\mathrm{G}$ before being digitized on the CCD.

The total number of counts $N_{T}$ in a Thomson scattering spectrum is proportional to the electron density $n_{e}$

$$
N_{T}=k \cdot n_{e} \frac{d \sigma_{T}}{d \Omega}
$$

where $d \sigma / d \Omega=r_{e}^{2}$ for scattering perpendicular to the probe beam, and $r_{e}=2.818 \times 10^{-15} \mathrm{~m}$ is the classical electron radius 2 . If $k$ is known, $n_{e}$ can be deduced from the measured counts. Since $k$ is difficult to calculate accurately a priori, it is easier to determine the constant in situ. This can be done using the relative signal intensities of Rayleigh ${ }^{26}$ or Raman 27 scattering off of a gas. Since stray light and Rayleigh scattered light have the same wavelength $\left(\lambda_{i}\right)$, measurements at different pressures and without the notch filter are needed to distinguish between Rayleigh scattered light and stray light. In this experiment, this calibration is performed using Raman scattering off of gaseous nitrogen at a pressure of $0.86 \mathrm{~atm}$. Raman scattering has the advantage of producing light outside the notch filter, over a wavelength range and at a signal intensity similar to TS. The disadvantage is that the signal is weak, so spectra must be averaged over thousands of shots to accumulate sufficient photon counts.

Fig. 3 a shows a raw Raman scattering CCD image averaged over 20,000 shots. The spectrum consists of a number of spectral peaks on both sides of the $532 \mathrm{~nm}$ laser-line, blocked by the notch (in the center of the image). Each peak corresponds to a transition from one rotational state to another, induced by the inelastic scattering process, where each state is characterized by the rotational integer quantum number $J$. The energy of a rotational state $J$ is given by $E_{J}=B \cdot J(J+1)$, where $B=2.48 \times 10^{-4} \mathrm{eV}$ for nitrogen ${ }^{28}$. Only transition $J \rightarrow J+2$

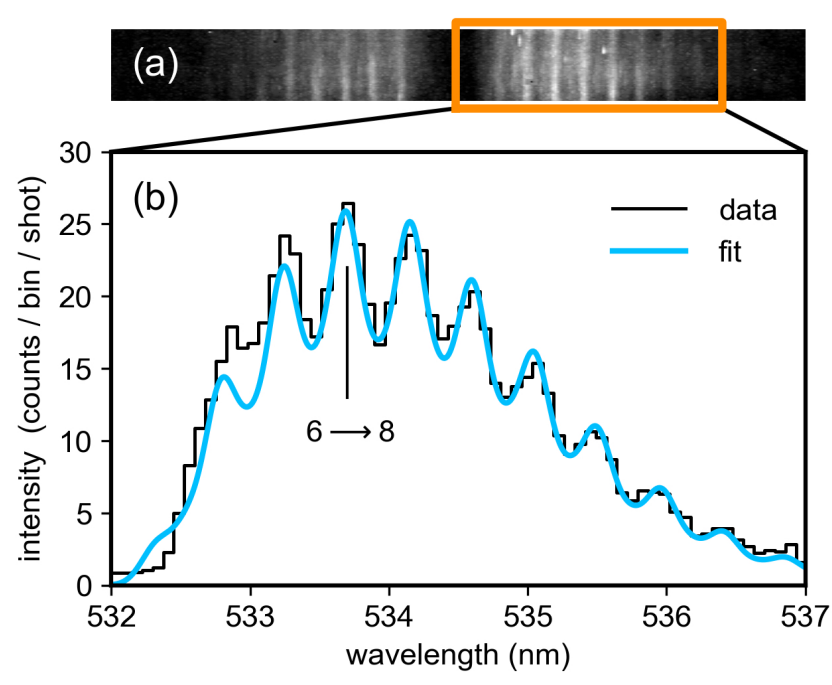

FIG. 3. (a) Raw CCD image of a Raman scattering spectrum showing several transition lines around the notch at $\lambda_{i}$ in the center. (b) Measured spectrum of the red-shifted Stokes lines (black) and comparison with the synthetic fit (blue). Only the bright even J-lines are separated at the spectrometer resolution. The brightest line is the $J=6 \rightarrow 8$ transition at $533.7 \mathrm{~nm}$. The area under the fit is used to determine $k$.

(Stokes) and $J \rightarrow J-2$ (anti-Stokes) are allowed. The wavelengths of the red-shifted Stokes lines may be approximated by 13

$$
\lambda_{J \rightarrow J+2} \approx \lambda_{i}+\frac{\lambda_{i}^{2}}{h c} B(4 J+6) .
$$

The total number of scattered photons in a single Raman line $J$ is given by

$$
N_{J \rightarrow J^{\prime}}=k \cdot n_{J} \frac{d \sigma_{J \rightarrow J^{\prime}}}{d \Omega},
$$

where the differential cross section $d \sigma / d \Omega$ varies with state $e^{13}$ around a weighted average of $3.8 \times 10^{-34} \mathrm{~m}^{2}$. The density $n_{J}$ of a rotational state $J$ is

$$
n_{J}=n_{g a s} \frac{g_{J}(2 J+1)}{Q} \exp \left(-\frac{E_{J}}{k_{B} T}\right),
$$

where the partition sum is $Q \approx 9 k_{B} T / B$, and $g_{J}$ is a statistical weight factor which is 6 or 3 for even or odd J respectively 29 . The counts in the Raman fine-structure spectrum as a function of wavelength is then

$$
I_{\mathrm{fs}}(\lambda)=k \sum_{J} n_{J} \frac{d \sigma_{J \rightarrow J^{\prime}}}{d \Omega} \delta\left(\lambda-\lambda_{J \rightarrow J^{\prime}}\right),
$$

where $\delta\left(\lambda-\lambda_{J \rightarrow J^{\prime}}\right)$ is the delta function. The width of the measured Raman peaks is determined by the instrument broadening, and the synthetic Raman spectrum $I_{\text {fit }}=I_{\mathrm{fs}} \circledast I_{\text {instr }}$ can be constructed via convolution of the fine structure profile and the experimentally measured instrument function $I_{\text {instr }}$. 
Fig. 3 shows the measured Stokes Raman spectrum and comparison with the synthetic fit. The spectral resolution only allows the brighter even J-lines to be separated. The total intensity of all Stokes lines is calculated from the area under the synthetic fit, since transition lines closest to the laser line are partially suppressed by the notch. Summing equation 5 over all Stokes J-lines visible at room temperature $(J<25)$ gives the total counts in the Stokes spectrum at density $N_{\text {gas }}$ from which $k$ can be determined.

$$
N_{\text {Stokes }}=k \cdot n_{\text {gas }} \cdot 3.82 \times 10^{-34} \mathrm{~m}^{2} .
$$

We measured a total Stokes signal of $745 \pm 50$ counts per shot for a gas density of $n_{\text {gas }}=(2.1 \pm 0.01) \times 10^{19} \mathrm{~cm}^{-3}$, corresponding to $k=(9.3 \pm 0.7) \times 10^{10} \mathrm{~m}$. The gas density was determined from a pressure measurement at room temperature using a capacitance manometer. Misalignment of the collection branch relative to the probe beam decreases the calibration factor $k$. However, accurate and reproducible alignment can be accomplished by automatically scanning the lens and fiber across the beam as shown in figure $1 \mathrm{~b}$ to find the peak. Alignment has been found to be stable throughout the day.

\section{RESULTS AND DISCUSSION}

Fig. 4 compares the TS spectra obtained for a $5 \mathrm{~J}$ heater beam at $y=15 \mathrm{~mm}$ and $20 \mathrm{~mm}$ from the target. The profiles are recorded at $150 \mathrm{~ns}$ and $225 \mathrm{~ns}$ respectively. Each spectrum is averaged over $400 \mathrm{TS}$ shots and an equal number of plasma-only background shots. The central bins of both spectra are suppressed by the notch at $\lambda_{i}$. Closer to the target, the spectrum exhibits a super-Gaussian profile with a width around $9 \mathrm{~nm}$ (FWHM). The spectrum further from the target has a smaller amplitude and area under the curve, a smaller width around $7 \mathrm{~nm}$ (FWHM), and a more Gaussian shape.

Thomson scattering can be collective or non-collective, depending on the dimensionless scattering parameter $\alpha=$ $1 /\left(k \lambda_{D}\right)$, where $k=4 \pi \cdot \sin (\theta / 2) / \lambda_{i}$ is the scattering vector and $\lambda_{D}=\sqrt{\varepsilon_{0} k_{B} T_{e} / n_{e} e^{2}}$ is the Debye length. When $\alpha \ll 1$, the scattering is incoherent (non-collective) and the spectrum reflects the Doppler shifted thermal electron motion, resulting in a Gaussian scattering spectrum. For $\alpha>1$, the scattering is coherent (collective), and the spectrum is influenced by the interactions between electrons and ions in the plasma. The spectrum will be non-Gaussian even if the underlying velocity distribution is Maxwellian as assumed here. The parameters in this experiment result in scattering parameters near unity and so the spectra range from weakly collective or non-collective with increasing distance from the targe ${ }^{1993031}$. In this experiment the ion feature is blocked by the notch, and the TS spectral density function $S(k, \omega)$ that describes the shape of the scattered spectrum is determined only by the electron component. Assuming a quasi-neutral plasma and Maxwellian velocity distribution for the electrons with thermal speed $v_{T e}$, the spectral density function is given by ${ }^{2}$

$$
S(k, \omega)=\frac{2 \sqrt{\pi}}{k v_{T e}}\left|1-\frac{\chi_{e}}{\varepsilon}\right|^{2} \exp \left(-\frac{\omega^{2}}{\left(k v_{T e}\right)^{2}}\right),
$$

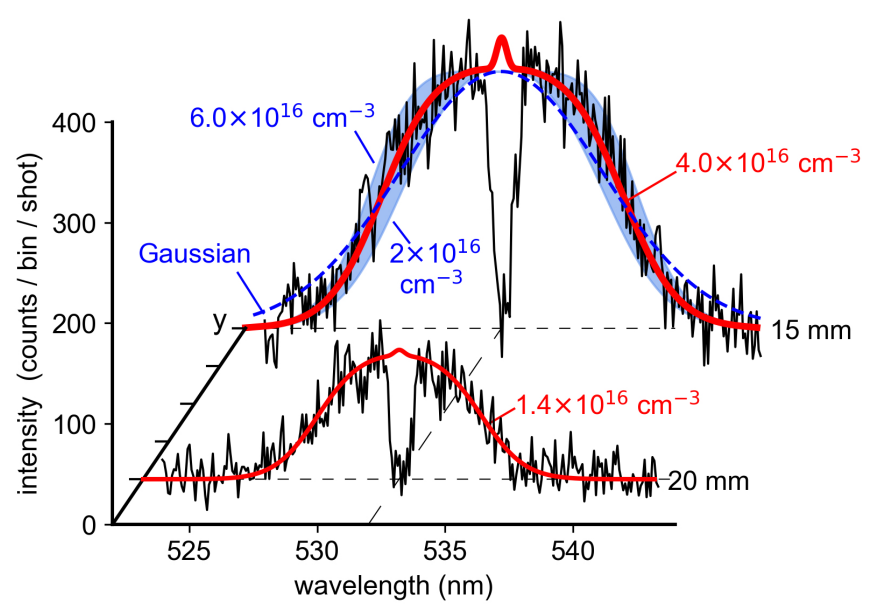

FIG. 4. Measured TS spectra at $y=15 \mathrm{~mm}$ at $150 \mathrm{~ns}$ and $y=$ $20 \mathrm{~mm}$ at $\mathrm{t}=225 \mathrm{~ns}$, with their central bins suppressed by the notch. The $15 \mathrm{~mm}$ spectrum is clearly super-Gaussian and can only be fit with a weakly collective spectrum for $n_{e}=4 \times 10^{16} \mathrm{~cm}^{-3}$ and $T_{e}=$ $7 \mathrm{eV}$. The upper and lower edge of the blue shaded curve show the collective fit for $6 \times 10^{16} \mathrm{~cm}^{-3}$ and $2 \times 10^{16} \mathrm{~cm}^{-3}$, respectively. The $20 \mathrm{~mm}$ spectrum is markedly lower in amplitude and exhibits a more Gaussian spectrum consistent with $n_{e}=1.4 \times 10^{16} \mathrm{~cm}^{-3}$ and $T_{e}=$ $4 \mathrm{eV}$.

where $\varepsilon=1+\chi_{e}+\chi_{i}$ is the plasma dielectric function, $\chi_{e}$ and $\chi_{i}$ are the electron and ion susceptibilities, respectively, $\omega=\omega_{s}-\omega_{i}, \omega_{i}$ is the probe beam frequency, and $\omega_{s}$ is the frequency of the scattered light. By fitting the experimental spectra with Eq. 9 convolved with the instrument function, the plasma parameters and $\alpha$ can be inferred. The broad shoulders in the $15 \mathrm{~mm}$ spectrum in Fig. 4 can only be fitted accurately for temperatures in the range $T_{e}=(7 \pm 0.5) \mathrm{eV}$ and densities in the range $(4 \pm 2) \times 10^{16} \mathrm{~cm}^{-3}$, as indicated by the blue shaded area, equivalent to $\alpha=0.61$. In this regime the fit to the weakly collective spectrum serves as an independent density diagnostic. The total number of counts in the measured TS spectrum (including those suppressed by the notch) is $N_{T}=(3.3 \pm 0.2) \times 10^{4}$, estimated by calculating the area under the fit which includes the region suppressed by the notch. Using the experimental throughput parameter $k$ measured from the Raman spectrum, this count is equivalent to a density of $n_{e}=(4.5 \pm 0.6) \times 10^{16} \mathrm{~cm}^{-3}$, which agrees with the density determined from the collective fit within $10 \%$. Farther from the target, the fit to the spectrum only yields the temperature, and the density must be determined from the area under the TS spectrum. The fit to the profile at $20 \mathrm{~mm}$ in Fig. 4 corresponds to $1.4 \times 10^{16} \mathrm{~cm}^{-3}, T_{e}=4 \mathrm{eV}$, and $\alpha=0.44$.

Fig. 5 shows the plasma parameters as a function of distance from the target at $t=150 \mathrm{~ns}$ (a), and as a function of time at $y=15 \mathrm{~mm}(\mathrm{~b})$. This data was recorded automatically by the data-acquisition system in more than $10^{4}$ laser shots over the course of four hours, by translating the scattering volume or delaying the probe pulse. Both the density and temperature fall off nearly linearly with distance from the target, consistent with an isentropic fluid mode ${ }^{6}$. Since the density falls off faster with distance from target than the temperature, 


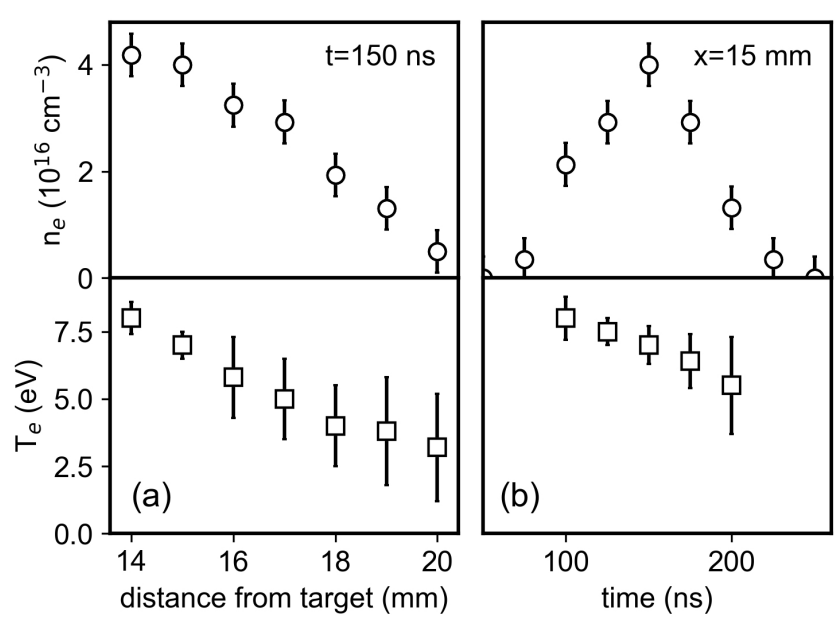

FIG. 5. (a) Electron density and temperature as a functions of distance from the target, $150 \mathrm{~ns}$ after the heater pulse. (b) Density and temperature as a function of time after the heater pulse at a fixed distance of $y=15 \mathrm{~mm}$ from the target. The data was obtained by scanning the scattering volume automatically along the probe beam.

the scattering parameter $\alpha \sim \sqrt{n_{e} / T_{e}}$ also decreases with distance. Data closer to the target than $\sim 12 \mathrm{~mm}$ was dominated by intense plasma emission lines (not shown), including neutral carbon lines at $526.9 \mathrm{~nm}$ and $528.8 \mathrm{~nm}$, with amplitudes of up to $2 \times 10^{3}$ counts and several times higher than the TS signal. The increased shot noise makes it challenging to fit to the TS signal for these spectra.

At a distance of $15 \mathrm{~mm}$ from the target the peak plasma density arrives $150 \mathrm{~ns}$ after the heater pulse (Fig. 5b), consistent with a bulk plasma flow velocity of $100 \mathrm{~km} / \mathrm{s}$. Scattered light can be detected as early as $75 \mathrm{~ns}$ after the heater beam, so the leading edge of the plasma moves at least at $200 \mathrm{~km} / \mathrm{s}$, consistent with previous spectroscopic measurements ${ }^{32}$. Scattering spectra in LPPs produced with a higher-energy $10 \mathrm{~J}$ heater beam show slightly higher densities and temperatures as expected 6 . At a distance of $20 \mathrm{~mm}$ from the target at 225 ns we measured a density and temperature of $1.7 \times 10^{16} \mathrm{~cm}^{-3}$ and $6 \mathrm{eV}$, respectively. Closer to the target, at $15 \mathrm{~mm}$, the $10 \mathrm{~J}$ spectra at $150 \mathrm{~ns}$ are dominated by plasma emission lines (not shown), but the TS fit is consistent with $n_{e}=(5.5 \pm 1) \times$ $10^{16} \mathrm{~cm}^{-3}$ and $T_{e}=10 \pm 2 \mathrm{eV}$.

\section{SUMMARY}

We have developed a high-repetition rate Thomson scattering diagnostic that allows auto-alignment and translation of the scattering volume over an arbitrary shaped spatial pattern, as the LPP is produced repeatedly at $1 \mathrm{~Hz}$ for thousands of shots. Using this technique we have measured $n_{e}$ and $T_{e}$ in an exploding laser plasma at distances of several centimeters from the target with spatial and temporal resolution. Unshifted stray light due to probe beam scattering off optics and surfaces inside the vessel has been effectively mitigated using a triple-grating-spectrometer and notch filter with an ex- tinction ratio of $10^{5}$. These measurements demonstrate that a collection branch design that sacrifices optical extent at the source in order to reduce beam pointing sensitivity makes it possible to automatically translate the scattering volume using motorized stages without the need for realignment. Electron densities obtained by fitting the data with the weakly collective spectral density function agree well with densities obtained from an absolute irradiance calibration via Raman scattering.

These first proof-of-concept measurements were hampered by the very low probe beam energy $(50 \mathrm{~mJ})$, which results in a TS signal close to the detection limit for these plasma parameters. Near the target or in plasmas produced with a $10 \mathrm{~J}$ heater beam, the spectra are dominated by carbon emission lines with intensities five times the TS signal. Scattering spectra are therefore averaged over hundreds of laser shots for each spatial position to improve SNR. A future iteration of this diagnostic will use a higher energy probe beam $\left(E_{\text {pulse }}=0.7 \mathrm{~J}\right)$ to increase the number of TS photons while keeping the plasma emission background constant. Per Eq. 1 this would increase the SNR by more than a factor of 5 for the case $N_{T}=N_{b g}$. In spectra where the plasma emission now exceeds the TS signal $\left(N_{b g}=5 \cdot N_{T}\right)$, the SNR would increase by a factor of 8. TS spectra can then be collected at these densities in as few as 10-20 laser shots. In combination with an additional stepper-controlled stage to translate the final probe-beam turning mirror along the $\hat{x}$ axis, this technique will allow point TS measurements in laser plasmas on a two-dimensional grid for the first time, and produce 2D density and temperature maps. While the current low probe beam energy requires too many shots to feasibly perform 2D scans, this limitation will be rectified with a higher energy laser.

\section{DATA AVAILABILITY STATEMENT}

The data that support the findings of this study are available from the corresponding author upon reasonable request.

\section{ACKNOWLEDGMENTS}

This work was supported by the Department of Energy under contract numbers DE-SC0019011 and DE-SC0021133, by the Defense Threat Reduction Agency and Lawrence Livermore National Security LLC under contract number B643014, by the National Nuclear Security Administration Center for Matter Under Extreme Conditions under award number DENA0003842, and by the National Science Foundation Graduate Fellowship Research Program under award number DGE1650604. We thank NIWC Pacific and Curtiss-Wright MIC for help with the slab laser system.

\footnotetext{
${ }^{1}$ S. H. Glenzer, W. E. Alley, K. G. Estabrook, J. S. De Groot, M. G. Haines, J. H. Hammer, J.-P. Jadaud, B. J. MacGowan, J. D. Moody, W. Rozmus, L. J. Suter, T. L. Weiland, and E. A. Williams. Thomson scattering from laser plasmas. Physics of Plasmas, 6(5):2117-2128, 1999. doi: 10.1063/1.873499 URL https://doi.org/10.1063/1.873499
} 
${ }^{2}$ D.H. Froula, S.H. Glenzer, N.C. Luhmann Jr., J. Sheffield, and T.J.H. Donné (Reviewer). Plasma scattering of electromagnetic radiation: Theory and measurement techniques. Fusion Science and Technology, 61: 104-105, 2012.

${ }^{3}$ C. Niemann, W. Gekelman, C. G. Constantin, E. T. Everson, D. B. Schaeffer, A. S. Bondarenko, S. E. Clark, D. Winske, S. Vincena, B. Van Compernolle, and P. Pribyl. Observation of collisionless shocks in a large current-free laboratory plasma. Geophysical Research Letters, 41(21): 7413-7418, 2014. ISSN 1944-8007. doi:10.1002/2014GL061820 URL http://dx.doi.org/10.1002/2014GL061820

${ }^{4}$ J.N. Leboeuf, K.R. Chen, J.M. Donato, D.B. Geohegan, C.L. Liu, A.A. Puretzky, and R.F. Wood. Modeling of plume dynamics in laser ablation processes for thin film deposition of materials. Physics of Plasmas, 3(5): 2203-2209, 1996. doi:10.1063/1.871676 URL https://doi.org/10. $1063 / 1.871676$

${ }^{5}$ C. Phipps, M. Birkan, W. Bohn, H.A. Eckel, H. Horisawa, T. Lippert, M. Michaelis, Y. Rezunkov, A. Sasoh, W. Schall, S. Scharring, and J. Sinko. Review: Laser-ablation propulsion. Journal of Propulsion and Power, 26 (4):609-637, 2010. doi:10.2514/1.43733 URL https://doi.org/10. $2514 / 1.43733$

${ }^{6}$ D.B. Schaeffer, A.S. Bondarenko, E.T. Everson, S.E. Clark, C.G. Constantin, and C. Niemann. Characterization of laser-produced carbon plasmas relevant to laboratory astrophysics. Journal of Applied Physics, 120(4), 2016. doi:http://dx.doi.org/10.1063/1.4959148 URL http://scitation.aip.org/content/aip/journal/jap/120/4/ 10.1063/1.4959148

${ }^{7}$ P.V. Heuer, M.S. Weidl, R.S. Dorst, D.B. Schaeffer, S.K.P. Tripathi, S. Vincena, C.G. Constantin, C. Niemann, L.B. Wilson III, and D. Winske. Laboratory observations of ultra-low-frequency analog waves driven by the right-hand resonant ion beam instability. The Astrophysical Journal, 891 (1):L11, feb 2020. doi:10.3847/2041-8213/ab75f4 URL https://doi. org/10.3847/2041-8213/ab75f 4

${ }^{\gamma}$ N. Peacock, D. Robinson, and M. Forrest. Measurement of the electron temperature by Thomson scattering in tokamak T3. Nature, 224:488-490, 1969.

${ }^{9}$ H. Murmann, S. Götsh, H. Röhr, H. Salzmann, and K.H. Steuer. The thomson scattering systems of the asdex upgrade tokamak. Rev. Sci. Instrum., 63 (10):4941, 1992.

${ }^{10}$ D. Johnson, N. Bretz, B. LeBlanc, R. Palladino, D. Long, and R. Parsells. Multipulse thomson scattering system for the national spherical torus experiment. Rev. Sci. Instrum., 70(1):776, 1999.

${ }^{11}$ B. P. LeBlanc. Thomson scattering density calibration by rayleigh and rotational raman scattering on nstx. Review of Scientific Instruments, 79 (10):10E737, 2008. doi:10.1063/1.2956747 URL https://doi.org/ 10.1063/1.2956747

${ }^{12}$ J.M. de Regt, R.A.H. Engeln, F.P.J. de Groote, J.A.M. van der Mullen, and D.C. Schram. Thomson scattering experiments on a $100 \mathrm{MHz}$ inductively coupled plasma calibrated by Raman scattering. Rev. Sci. Instrum., 66: 3228, 1995.

${ }^{13}$ M.J. Van de Sande. Laser scattering on low temperature plasmas : high resolution and stray light rejection. PhD thesis, Eindhoven, 2002. https://doi.org/10.6100/IR554040.

${ }^{14}$ A. Kono and K. Nakatani. Efficient multichannel thomson scattering measurement system for diagnostics of low-temperature plasmas. Review of Scientific Instruments, 71(7):2716-2721, 2000. doi:10.1063/1.1150680 URL https://doi.org/10.1063/1.1150680

${ }^{15}$ H. J. van der Meiden, R. S. Al, C. J. Barth, A. J. H. Donné, R. Engeln, W. J. Goedheer, B. de Groot, A. W. Kleyn, W. R. Koppers, N. J. Lopes Cardozo, M. J. van de Pol, P. R. Prins, D. C. Schram, A. E. Shumack, P. H. M. Smeets, W. A. J. Vijvers, J. Westerhout, G. M. Wright, and G. J. van Rooij. High sensitivity imaging thomson scattering for low temperature plasma. Review of Scientific Instruments, 79(1):013505, 2008. doi:10.1063/1.2832333

${ }^{16}$ Emile Carbone and Sander Nijdam. Thomson scattering on nonequilibrium low density plasmas: principles, practice and challenges. Plasma Physics and Controlled Fusion, 57(1):014026, nov 2014. doi: 10.1088/0741-3335/57/1/014026 URL https://doi.org/10.1088/ 0741-3335/57/1/014026

${ }^{17}$ D.B. Schaeffer, D.S. Montgomery, A.S. Bondarenko, L.A. Morton, R.P. Johnson, T. Shimada, C.G. Constantin, E.T. Everson, S.A. Letzring, S.A. Gaillard, K.A. Flippo, S.H. Glenzer, and C. Niemann. Thomson scatter- ing measurements of temperature and density in a low-density, laser-driven magnetized plasma. Journal of Instrumentation, 7(02):P02002, 2012.

${ }^{18}$ G. Gregori, S. H. Glenzer, J. Knight, C. Niemann, D. Price, D. H. Froula, M. J. Edwards, R. P. J. Town, A. Brantov, W. Rozmus, and V. Yu. Bychenkov. Effect of nonlocal transport on heat-wave propagation. Phys. Rev. Lett., 92:205006, May 2004. doi:10.1103/PhysRevLett.92.205006 URL https://link.aps.org/doi/10.1103/PhysRevLett.92.205006

${ }^{19}$ D.B. Schaeffer, C.G. Constantin, A.S. Bondarenko, E.T. Everson, and C. Niemann. Spatially resolved Thomson scattering measurements of the transition from the collective to the non-collective regime in a laserproduced plasma. Review of Scientific Instruments, 87(11), 2016. doi: http://dx.doi.org/10.1063/1.4955304

${ }^{20}$ W.S. Brocklesby, J. Nilsson, T. Schreiber, J. Limpert, A. Brignon, J. Bourderionnet, L. Lombard, V. Michau, M. Hanna, Y. Zaouter, T. Tajima, and G. Mourou. ICAN as a new laser paradigm for high energy, high average power femtosecond pulses. The European Physical Journal Special Topics, 223:1189-1195, 2014.

${ }^{21}$ S. Weber, S. Bechet, S. Borneis, L. Brabec, M. Bučka, E. Chacon-Golcher, M. Ciappina, M. DeMarco, A. Fajstavr, K. Falk, E.-R. Garcia, J. Grosz, Y.J. Gu, J.-C. Hernandez, M. Holec, P. Janečka, M. Jantač, M. Jirka, H. Kadlecova, D. Khikhlukha, O. Klimo, G. Korn, D. Kramer, D. Kumar, T. Lastovička, P. Lutoslawski, L. Morejon, V. Olšovcová, M. Rajdl, O. Renner, B. Rus, S. Singh, M. Šmid, M. Sokol, R. Versaci, R. Vrána, M. Vranic, J. Vyskočil, A. Wolf, and Q. Yu. P3: An installation for high-energy density plasma physics and ultra-high intensity laser-matter interaction at ELIbeamlines. Matter and Radiation at Extremes, 2(4):149-176, 2017. doi: 10.1016/j.mre.2017.03.003 URL https://aip.scitation.org/doi/ $\mathrm{abs} / 10.1016 / \mathrm{j} . \mathrm{mre} .2017 .03 .003$

${ }^{22}$ J. Nees, A. Maksimchuk, G. Kalinchenko, B. Hou, Y. Ma, P. Campbell, A. McKelvey, L. Willingale, I. Jovanovic, C. Kuranz, A. Thomas, and K. Krushelnick. ZEUS: A National Science Foundation mid-scale facility for laser-driven science in the QED regime. In Conference on Lasers and Electro-Optics, page JW2B.9. Optical Society of America, 2020. URL http://www.osapublishing.org/abstract.cfm?URI= CLEO_SI-2020- JW2B.9

${ }^{23}$ S. Meuren, D.A. Reis, R. Blandford, P.H. Bucksbaum, N.J. Fisch, F. Fiuza, E. Gerstmayr, S. Glenzer, M.J. Hogan, C. Pellegrini, M.E. Peskin, K. Qu, G. White, and V. Yakimenko. Research opportunities enabled by colocating multi-petawatt lasers with dense ultra-relativistic electron beams. arXiv, 2105.11607, 2021.

${ }^{24}$ C.B. Dane, L.E. Zapata, W.A. Neuman, M.A. Norton, and L.A. Hackel. Design and operation of a $150 \mathrm{~W}$ near diffraction-limited laser amplifier with SBS wavefront correction. IEEE Journal of Quantum Electronics, 31 (1), 1995.

${ }^{25}$ D.B. Schaeffer, L.R. Hofer, E.N. Knall, P.V. Heuer, C.G. Constantin, and C. Niemann. A platform for high-repetition-rate laser experiments on the Large Plasma Device. High Power Laser Science and Engineering, 6:e17, 2018.

${ }^{26}$ L.A. Berni, D.O. Campos, M. Machida, S.A. Moshkalyov, and S.V. Lebedev. Molecular Rayleigh scattering as calibration method for Thomson scattering experiments. Brazilian Journal of Physics, 26(4):755, 1996.

${ }^{27}$ F. Flora and L. Giudicotti. Complete calibration of a Thomson scattering spectrometer system by rotational Raman scattering in $\mathrm{H}_{2}$. Applied Optics, 26(18):4001, 1987.

${ }^{28}$ C.M. Penney, R.L. St. Peters, and M. Lapp. Absolute rotational Raman cross sections for $\mathrm{N}_{2}, \mathrm{O}_{2}$, and $\mathrm{CO}_{2}$. Journ. Opt. Soc. Am., 64:712, 1974.

${ }^{29} \mathrm{G}$. Herzberg. Molecular spectra and molecular structure volume I. Spectra of Diatomic Molecules. D. van Nostrand Company Inc., Princeton, 3 edition, 1950.

${ }^{30}$ J.S. Ross, S.H. Glenzer, J.P. Palastro, B.B. Pollock, D. Price, G.R. Tynan, and D.H. Froula. Thomson-scattering measurements in the collective and noncollective regimes in laser produced plasmas (invited). Review of Scientific Instruments, 81(10):10D523, 2010. doi:10.1063/1.3478975 URL https://doi.org/10.1063/1.3478975

${ }^{31}$ Byonghoon Seo and Paul M. Bellan. Experimental investigation of the compression and heating of an mhd-driven jet impacting a target cloud. Physics of Plasmas, 25(11):112703, 2018. doi:10.1063/1.5045678 URL https://doi.org/10.1063/1.5045678

${ }^{32}$ R.S. Dorst, P.V. Heuer, D.B. Schaeffer, C.G. Constantin, and C. Niemann. Measurements of ion velocity distributions in a large scale laser-produced 
